\title{
Design of a cross-dipole array acoustic logging tool
}

\author{
Lu Junqiang ${ }^{1}$, Ju Xiaodong ${ }^{*}$ and Cheng Xiangyang ${ }^{2}$ \\ ${ }^{1}$ School of Resources and Information Technology, China University of Petroleum, Beijing 102249, China \\ ${ }^{2}$ School of Petroleum Engineering, China University of Petroleum, Dongying, Shandong 257061, China
}

\begin{abstract}
When entering an anisotropic formation, a shear wave splits into a fast wave and a slow wave. Based on the principle of four-component cross-dipole acoustic wave measurement, the anisotropy of HTI (Horizontal Transverse Isotropy) formation can be determined. The method of calculating the fast and slow wave data when a shear wave propagates along the borehole axis in anisotropic formation was analyzed, and the implementation of a cross-dipole acoustic logging tool was demonstrated. The tool was composed of transmitter electronics, transmitter mandrel, acoustic isolator, receiver mandrel and main control electronics. Sonde, transmitter circuit, signal receiving and processing circuit, data acquisition system, system control circuit and telemetry interface circuit were presented and analyzed. The test model was used in production wells and standard wells in various areas and the four-component cross-dipole waves were acquired and processed. The waves had good signal-to-noise ratio and clear characteristics, and the fast and slow waveforms, processed slowness curves, anisotropy and fast shear wave azimuth well matched with each other.
\end{abstract}

Key words: Acoustic logging tool, cross-dipole, anisotropy, electronics, sonde

With the development of oil and gas exploration and exploitation, the problem of formation anisotropy is becoming increasingly prominent. By studying the anisotropy of formations, we can detect fractures and analyze the formation stress field, which is helpful for evaluationof fractured reservoir beds, borehole condition monitoring and guidance for hydraulic fracturing and acidizing ( $\mathrm{Lu}, 2007)$. Crossdipole array acoustic logging technology which can determine the shear wave by measuring the flexural wave overcomes the restriction that shear waves cannot be measured in soft formations (Qiao et al, 2006; Sun et al, 2007). The shear wave acquired can be used for studying the formation anisotropy (Tang and Cheng, 2004). This technology is widely used in petroleum exploration. Therefore, in order to meet the growing demand for exploration and exploitation of complex reservoirs, we must develop a Chinese proprietary acoustic logging tool for high performance cross-dipole logging with high performance (Ju et al, 2005).

\section{Basic theory of cross-dipole measurement}

Anisotropy can be analyzed by the simplified transverse isotropy model (TI) which is in general use in borehole acoustic logging (Du and Yang, 2003; Vestrum et al, 1999). The TI anisotropy model has a symmetry axis. In any direction perpendicular to this axis, the medium characteristics are the same. Two kinds of TI cases are often encountered in petroleum exploration. One case is that the symmetry axis of the TI formation coincides with the borehole axis. It is called vertical TI or VTI. The existence of VTI is

*Corresponding author. email: juxdong@cup.edu.cn

Received June 6, 2007 related to the sedimentary process, such as the formation of mudstone and shale. The other case is azimuth anisotropy and its symmetry axis is perpendicular to the borehole axis. The azimuth anisotropy for a vertical borehole is also called horizontal TI or HTI and the symmetry axis is horizontal. The HTI around a borehole can be caused by fractures parallel to the borehole axis or an unbalanced formation stress field such as in a sandstone formation (Zamora and Poirier, 1990).

Fig. 1 shows a diagram illustrating the four-component cross-dipole logging in HTI formation (Tang and Cheng, 2004). Four-component cross-dipole acoustic wave data are recorded during logging. The data contain two in-line components XX and YY and two cross-line components $\mathrm{XY}$ and YX. The first letter denotes the cross-dipole source orientation, and the second letter denotes the receiver orientation. Fast shear wave azimuth EMBED Equation.3 and anisotropy are determined according to four-component wave data.

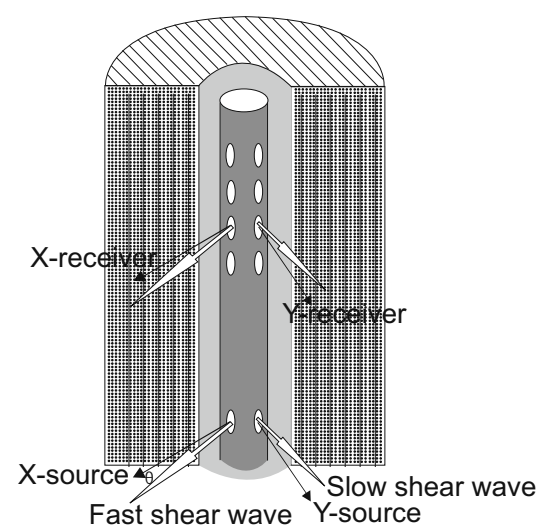

Fig.1 Four-component cross-dipole logging in HTI formation 


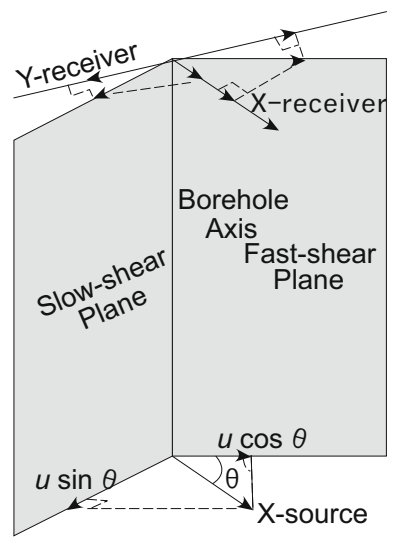

Fig.2 Shear wave splitting and calculation

The tool is assumed to be centered in the borehole. There is an angle $\theta$ between source-receiver plane in the $\mathrm{X}$ direction and fast shear wave polarization plane. When $\theta$ is non-zero, the shear wave splits into fast and slow shear waves, both of which propagate along the borehole axis and respectively polarize in fast and slow principal directions of HTI formation. In actual measurements, the source-receiver plane in the $\mathrm{X}$ direction is always not coincident with the fast shear wave polarization plane. When $\theta$ is $0^{\circ}$ or $90^{\circ}$, the polarization direction of the cross-dipole source coincides with the fast or slow shear wave plane, so only the fast or slow shear wave is excitated (Crampin, 1985; Olofsson and Probert, 2003).

Since the working theory in the $\mathrm{X}$ and $\mathrm{Y}$ directions is the same, we need only analyze the $\mathrm{X}$ direction. As shown in Fig. 2, a cross-dipole acoustic source in the $\mathrm{X}$ direction emits a wave signal $u$. Due to anisotropy, the wave will split into a fast shear wave $u \cos \theta$ and a slow shear wave $u \sin \theta$ when entering the formation. After propagating a distance from source to receiver, the fast and slow shear waves are $u^{*} g_{f} \cos \theta$ and $u^{*} g_{s} \sin \theta$ respectively, where $g_{f}$ and $g_{s}$ are the functions of fast and slow shear wave slowness. The XX and XY wave data are obtained by respectively projecting the fast and slow shear waves back to the $\mathrm{X}$ and $\mathrm{Y}$ axes:

$$
\left\{\begin{array}{l}
x x(t)=u^{*} g_{f} \cos ^{2} \theta+u^{*} g_{s} \sin ^{2} \theta \\
x y(t)=-u^{*} g_{f} \sin \theta \cos \theta+u^{*} g_{s} \sin \theta \cos \theta
\end{array}\right.
$$

The YY and YX wave data can be obtained by replacing $\theta$ with $\theta+90^{\circ}$ in the expression (1). According to above fourcomponent wave data, the fast and slow shear waves can be constructed, namely

$\left\{\begin{array}{l}F_{p}(t)=x x(t) \cos ^{2} \theta+[x y(t)+y x(t)] \sin \theta \cos \theta+y y(t) \sin ^{2} \theta \\ S_{p}(t)=x x(t) \sin ^{2} \theta-[x y(t)+y x(t)] \sin \theta \cos \theta+y y(t) \cos ^{2} \theta\end{array}\right.$

\section{Design of cross-dipole logging tool}

Cross-dipole logging is one of the functions of the multi-pole array acoustic logging tool (MPAL) and the implementation of cross-dipole measurement using the MPAL is analyzed ( $\mathrm{Lu}$ et al, 2007). The cross-dipole logging tool is composed of transmitter electronics, transmitter mandrel, acoustic isolator, receiver mandrel and main control electronics.

Fig. 3 shows the principle of the whole sonde which consists of transmitter transducers, acoustic isolator and receiver transducer array. Two perpendicular transmitter transducers located at the same depth and pulse transformer form the transmitter mandrel. The acoustic isolator is a mechanical attenuator which can isolate the acoustic wave energy in the whole frequency range, and attenuate and delay the direct interference signals effectively. The acoustic isolator's special structure makes it usable in a horizontal or deviated well. The receiver transducer array includes 8 sets of transducers, 32 units in total. Every set has two

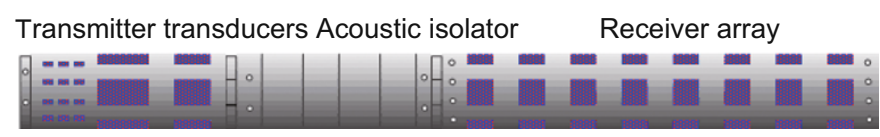

Fig. 3 Diagram illustrating acoustic logging sonde

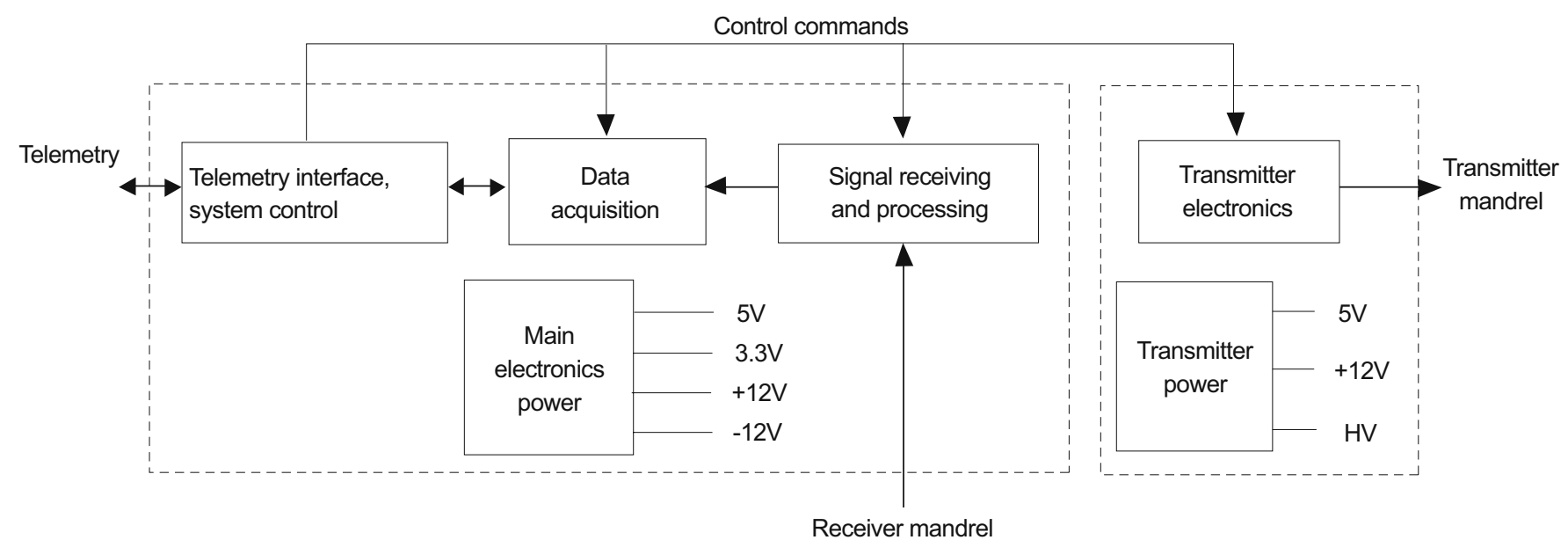

Fig. 4 Schematic electronics diagram 
pairs of transducers. One pair is in the same direction as the $\mathrm{X}$-transmitter, and the other pair is in the same direction as the Y-transmitter. All of them can receive in-line and crossline dipole signals. After processing and combining all the output signals with the receiver electronics, we can obtain the corresponding acoustic wave signals (Cheng et al, 2007).

The electronics consist mainly of the transmitter circuit, signal receiving and processing circuit, data acquisition circuit, system control circuit, telemetry interface circuit and power circuit. System control electronics controls all other circuits by special control commands (Ju et al, 2005). The functional diagram is shown in Fig. 4.

The transmitter electronics are located in the lowest part of the tool. It is composed of the control command receiving logic circuit, voltage conversion circuit, transmitter control circuit, power storage circuit and high-power pulse driver circuit of vertical metal oxide semiconductor (VMOS). The X-transducer or Y-transducer is activated by control commands and then a cross-dipole pulse at $1,800 \mathrm{~V}$ and with a variable width can be obtained from the pulse transformer, which has its own power supply of low and high voltage direct current.

Signal receiving and processing electronics process the acoustic waveform signals of 32 traces from the receiving transducer array, and dipole signals of a 8 traces are generated. The electronics is composed of a buffer amplifier, signal generator, signal selecting switchers, programmable gain control and band-pass filter. Two circuit channels will be needed if cross-dipole signals of a set of receiving transducer array are simultaneously processed, and the signals of adjacent receiving transducer array need not be processed. To handle a wide dynamic range of acoustic wave signals, a programmable gain amplifier (PGA) with 69db dynamic range is used. The PGA can be adjusted individually as well as synchronously in steps of $3 \mathrm{db}$. Frequency range of the band-pass filter is from $260 \mathrm{~Hz}$ to $20 \mathrm{kHz}$. Aliasing noise can be decreased by a higher order low-pass filter.

Synchronous and parallel acquisition of 8 channel waveforms with high speed and high precision is realized by data acquisition circuits and the acquired data are stored in first-in/first-out (FIFO) memory. A 14bit successiveapproximation analog-to-digital converter (ADC) with the maximum sampling velocity of $400 \mathrm{kSPS}$ is used. A complex programmable logic device (CPLD) acts as the acquisition controller and acquisition rate and acquisition depth can be dynamically set as needed by system control commands. After the system controller sends the commands, all the acquisition control procedure is automatically performed by the CPLD, which can save the system controller's resources (Lu et al, 2006; 2007).

System control and telemetry interface circuits, which are the control and transmission center of the tool, are based

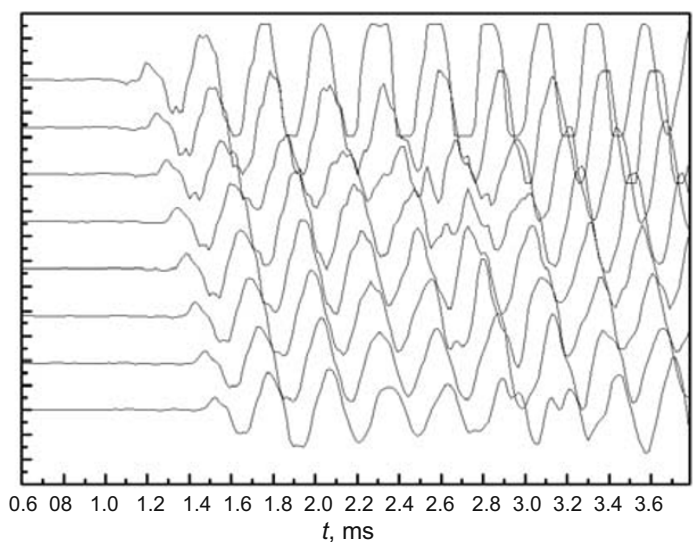

(a) $X X$

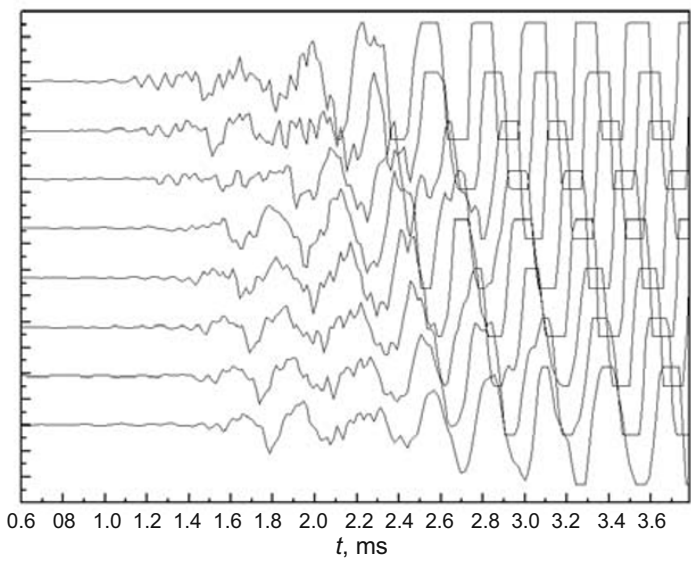

(c) $Y X$

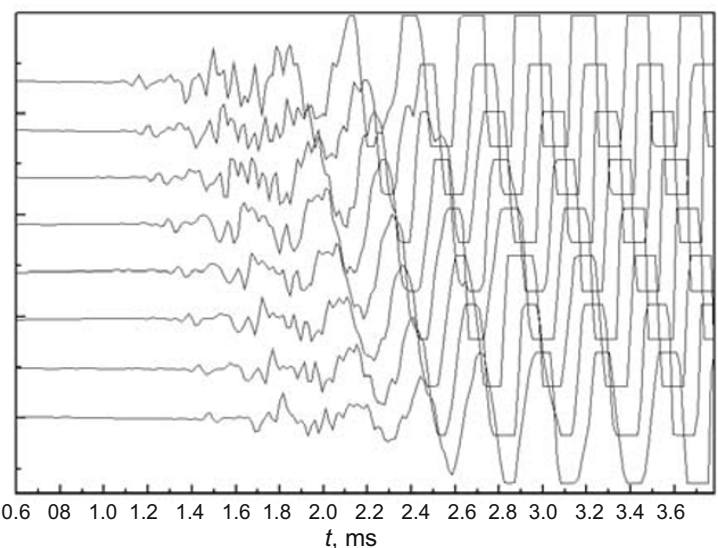

(b) $X Y$

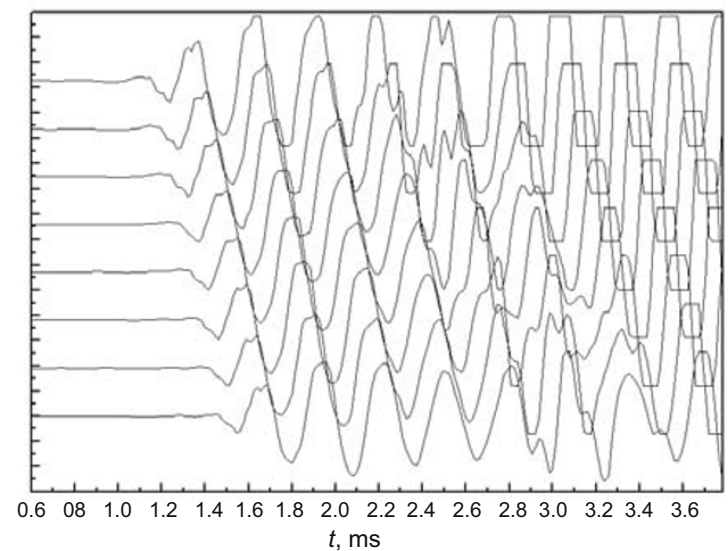

(d) $Y Y$

Fig. 5 Four-component dipole waveforms from an open borehole 
on a 16bit fixed point digital signal processor (DSP). A telemetry interface receives commands from surface, and uploads acquired data upon demand. The system control decodes received commands and performs the commands corresponding to acquisition modes. When receiving new an acquisition commands driven by depth interrupt, an acquisition cycle is started and the timing sequence and parameters of the signal receiving and processing circuit, acquisition circuit and transmitter circuit are set. After the acquisition cycle is finished, the resultant data are read from the data acquisition circuit, processed by system control circuit and then temporarily stored in random access memory (RAM). The DSP offers high processing speed, and the acquisition precision and signal-to-noise (SNR) can be improved by digital filtering, extracting and stacking for the wave data.

\section{Analysis of field testing results and conclusions}

The model tool was tested many times in test wells and production wells in various areas, and reliable logging data with good SNR and clear waveform characteristics were acquired in various receiving modes. In the test, we also validated the repeatability and consistency of testing using the logging tool. Fig. 5 shows four-component dipole waves acquired from an open borehole of production well at the corresponding depth of XX20.82m. It is shown that the waveform SNR of every component was satisfactory. The two cross-line components $\mathrm{XY}$ and $\mathrm{YX}$ have about $12 \mathrm{db}$ gain higher than the in-line components XX and YY. It can be seen clearly that the dipole signals of in-line components are stronger than that of cross-line components.

An important application of four-component cross-dipole logging is the analysis of formation anisotropy (Thomsen, 1986; Dillen et al, 1999). The extent of anisotropy can be evaluated according to the characteristics of fast and slow shear waves. Fig. 6 shows the analysis results of crossdipole data from an open hole of the same well in Fig. 5. Track 2 displays the curves of gamma-ray and azimuth of the logging tool. Track 3 shows the fast (DTSF) and slow (DTSS)

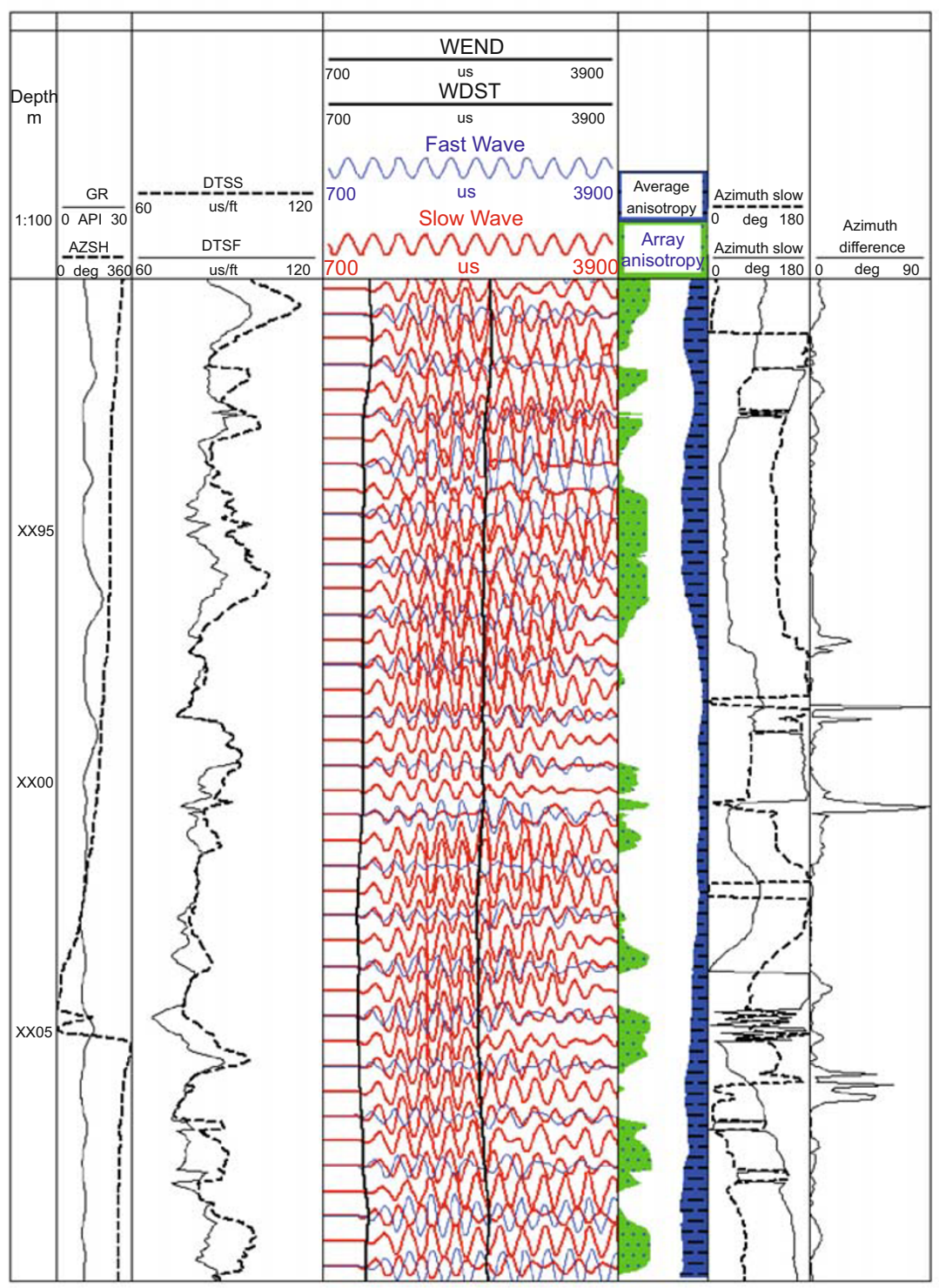

Fig. 6 Anisotropy analysis results of four-component cross-dipole data 
slowness curves which are expressed by continuous and broken curves respectively. Track 4 plots the fast and slow shear waves acquired by inversion and processing window. Track 5 shows the average anisotropy from transmitter to receiver (left to right) and the anisotropy over the receiver array aperture (right to left). The horizontal coordinates is $0-30 \%$. Track 6 plots the fast and slow shear wave azimuth curves. The right track shows the azimuth difference between the fast and the slow shear wave azimuth when the fast shear wave azimuth is plused $90^{\circ}$.

It can be seen from Fig. 6 that the initial portions of the fast and slow shear waveforms coincide and the corresponding formation anisotropy is close to 0 , when the fast and slow shear wave slowness curves coincide. Conversely, when the fast and slow shear wave slowness curves do not coincide, the initial portions of the fast and slow waveforms have some phase difference and the formation should be anisotropic. The azimuth difference in the far right track is almost zero, so the fast and slow shear wave azimuth values calculated are correct and reliable. When crossing the high-anisotropy formation, shear wave splitting can be clearly observed and the splitting into fast and slow shear waves starts at the initial portion of the waveforms.

\section{References}

Crampin S. Evaluation of anisotropy by shear-wave splitting. Geophysics. 1985. 50(1): 142-152

Cheng X Y, Ju X D, Lu J Q, et al. The design of a downhole multipole acoustic transducer transmitter with high power. Journal of China University of Petroleum. 2007. 31(6): 40-43 (in Chinese)

Dillen M W P, Cruts H M A, Groenenboom J, et al. Ultrasonic velocity and shear-wave splitting behavior of a Colton sandstone under a changing triaxial stress. Geophysics. 1999. 64(5): 1603-1607
Du Q Z and Yang H Z. Detection method for fractures in azimuthally anisotropic media. Journal of University of Petroleum. 2003. 27(4): 32-36 (in Chinese)

Ju X D, Cheng X Y, Lu J Q, et al. The design of downhole control command circuit based on CPLD and its application. Well Logging Technology. 2005. 29(4): 356-358 (in Chinese)

$\mathrm{Lu} \mathrm{J}$ Q. Electronic system research and designing of multi-pole array acoustic logging tool. Beijing: Ph.D. Dissertation, China University of Petroleum. 2007 (in Chinese)

Lu J Q, Ju X D and Cheng X Y. Research into multi-channel waveform data acquisition and processing used for cross dipole array acoustic logging. Journal of Oil and Gas Technology. 2007. 29(1): 74-77 (in Chinese)

Lu J Q, Ju X D, Cheng X Y, et al. Application research of high-speed and high-precision ADC in high temperature downholes. Computer Measurement and Control. 2006. 14(6): 754-756 (in Chinese)

Olofsson B and Probert T. Azimuthal anisotropy from the Valhall 4C 3D survey. The Leading Edge. 2003. 22(12): 1228-1235

Qiao W X, Ju X D, Che X H, et al. Multipole acoustic logging transmitter structurized by annular array. Journal of China University of Petroleum. 2006. 30(5): 33-41 (in Chinese)

Sun Z F, Qiao W X, Che X H. Optimization design of amplitude weighting for linear phased array transmitters in acoustic well logging. Journal of China University of Petroleum. 2007. 31(1): 40-44 (in Chinese)

Tang X M and Cheng A. Quantitative borehole acoustic methods. Amsterdam: Elsevier. 2004

Thomsen L. Weak elastic anisotropy. Geophysics. 1986. 51(10): 1239-1246

Vestrum R W, Lawton D C, Schmid R. Imaging structures below dipping TI media. Geophysics. 1999. 64(4): 1239-1246

Zamora M and Poirier J P. Experimental study of acoustic anisotropy and birefringence in dry and saturated Fontainebleau sandstone. Geophysics. 1990. 55(11): 1455-1465

(Edited by Yang Lei) 